\title{
CORRESPONDENCE
}

\section{Diet and depression: future needs to unlock the potential}

(c) The Author(s), under exclusive licence to Springer Nature Limited 2021

Molecular Psychiatry; https://doi.org/10.1038/s41380-021-01360-2

\section{TO THE EDITOR:}

In line with our previous responses to Dr Molendijk's correspondences (e.g., ref. [1]), we reassert that, although there are limitations in the current literature that require further investigation (as highlighted in our review and in previous work in the field), both observational and randomized controlled trial (RCT) data, supported by extensive preclinical data, are supportive of a role for diet in the aetiology and adjunctive treatment of depression. Indeed, Dr Molendijk and colleagues' own metaanalysis of prospective studies shows a $0.64-0.78$ odds ratio reduced risk of depressive symptoms with improved diet quality. Although their subgroup analysis of 3 (of 17) effect sizes reported no significant effect when controlling for baseline depressive symptoms, this is in contrast to a more recent meta-analysis that used a rigorous harmonized analysis to address between-study heterogeneity [2].

Regarding the emerging RCT data, findings of meta-analyses suggest a small but significant effect of dietary interventions on depressive symptoms in largely non-clinical populations (see ref. [3] and Thomas-Odenthal et al. 2020, cited in the original letter). Moreover, three existing RCTs in those with current depression show evidence of efficacy from dietary interventions [4-6], along with relationships between degree of dietary change and mood improvements, and-where investigated-cost-effectiveness due to benefits that extend beyond symptom improvement (e.g., see ref. [7]). With regards to the prevention of incident depression, the results of the most recent and largest study on this are consistent with a prevention effect. To explain: despite the 2019 MooDFOOD trial reporting a null result in their primary outcome, possibly owing to the lower-than-expected incident depression cases and a minimal average change in diet, the trial reported a reduction in the risk for incident depression in people who were classified as adherent to the dietary 'food-related behavioural activation therapy' intervention, an effect that was more pronounced in those with higher depressive symptoms at baseline [8]. In addition, a recent analysis of the MoodFood trial suggests that the dietary intervention may have also improved somatic and energy-related profiles of depressive symptoms [9]. Furthermore, although also limited by lower-than-expected transition to incident depression, a second prevention study showed that coaching to improve dietary habits was as efficacious as problem-solving therapy in reducing the transition to case-level depression in older Americans, with benefits that were sustained over 2 years [10]. Given Dr. Molendijk and colleagues' previous comments regarding expectation bias as the explanation for the positive findings in our SMILES trial [1], it is notable that the nutrition education intervention in this study was in fact the control condition and there was no expectation on the part of the investigators that dietary change would have any impact on depression outcomes.

It should also be emphasized that the cited limitations in Dr. Molendijk and colleagues' letter speak to the complexity of conducting non-pharmacological interventions more generally. Indeed, due to the nature of lifestyle interventions, whether it is dietary, physical activity, or otherwise, it is inherently difficult to incorporate clinical trial features such as double-blinding or placebo controls compared to pharmacotherapy interventions. Investigating mental health outcomes such as depression further compounds these challenges, as there are currently no objective biomarkers to measure clinical outcomes, necessitating a reliance on subjective measures. The inherent methodological challenges of nutrition research are also of relevance here. Partly for these reasons, we are currently conducting large-scale pragmatic trials to ascertain 'real-world' effectiveness and cost-effectiveness of dietary interventions in mental health. Given the focus of our review, the limitations of the existing diet-depression evidence base were not expanded upon; however, these have been addressed in other publications (e.g., see ref. [11]).

Given the growing evidence base regarding efficacy and costeffectiveness, and the increasing recognition of the importance of addressing diet and other lifestyle behaviours in mental health treatment, now reflected in official Australian clinical treatment guidelines (Mahli et al. [12]), it is entirely appropriate to posit how diet may modulate depression. To this end, the current review provided a detailed overview of selected mechanisms of action that are implicated in mental health and where the existing data suggest that they may be modulated by dietary intervention. We do not suggest that the data are unequivocal regarding the mechanisms of actions by which diet may exert its influence on mental health outcomes, because no clinical trial has been powered to examine this but rather, and as we have stated in our review, these are proposed mechanisms that require further investigation and this requires large-scale RCTs that facilitate their examination.

Wolfgang Marx (D ${ }^{1 凶}$, Melissa M. Lane ${ }^{1}$, Meghan Hockey ${ }^{1}$, Hajara Aslam ${ }^{1}$, Ken Walder ${ }^{2}$, Alessandra Borsini (D) $^{3}$, Joseph Firth (D) ${ }^{4,5}$, Carmine M. Pariante $\mathbb{D D}^{3}$, Kirsten Berding ${ }^{6}$, John F. Cryan (D) ${ }^{6,7}$, Gerard Clarke (D) ${ }^{6,8,9}$, Jeffrey M. Craig ${ }^{2}$, Kuan-Pin $\mathrm{Su}^{10,11,12}$, David Mischoulon ${ }^{13,14}$ Fernando Gomez-Pinilla ${ }^{15}$, Jane A. Foster ${ }^{16}$, Patrice D. Cani (D ${ }^{17}$, Sandrine Thuret $\mathbb{I D}^{18}$, Heidi M. Staudacher ${ }^{1}$, Almudena Sánchez-Villegas ${ }^{19,20}$, Husnain Arshad ${ }^{21}$, Tasnime Akbaraly (iD ${ }^{21,22}$, Adrienne $\mathrm{O}^{\prime}$ Neil $^{1}$ and Felice N. Jacka ${ }^{1,23,24,25}$

${ }^{1}$ IMPACT (The Institute for Mental and Physical Health and Clinical Translation), Food and Mood Centre, Deakin University, Geelong, VIC, Australia. ${ }^{2}$ IMPACT (The Institute for Mental and Physical Health and Clinical Translation), Metabolic Research Unit, Deakin University, Geelong, VIC, Australia. ${ }^{3}$ Department of Psychological Medicine, Institute of Psychiatry, Psychology and Neuroscience, King's College London, London, UK. ${ }^{4}$ Division of Psychology and Mental Health, 
University of Manchester, Manchester, UK. ${ }^{5}$ NICM Health Research Institute, Western Sydney University, Westmead, NSW, Australia. ${ }^{6}$ APC Microbiome Ireland, University College Cork, Cork, Ireland. ${ }^{7}$ Department of Anatomy and Neuroscience, University College Cork, Cork, Ireland. ${ }^{8}$ Department of Psychiatry and Neurobehavioural Science, University College Cork, Cork, Ireland. ${ }^{9}$ INFANT Research Centre, University College Cork, Cork, Ireland. ${ }^{10}$ Departments of Psychiatry and Mind-Body Interface Laboratory (MBI-Lab), China Medical University Hospital, Taichung, Taiwan. ${ }^{11}$ An-Nan Hospital, China Medical University, Tainan, Taiwan. ${ }^{12}$ College of Medicine, China Medical University, Taichung, Taiwan. ${ }^{13}$ Depression Clinical and Research Program, Department of Psychiatry, Massachusetts General Hospital, Boston, MA, USA. ${ }^{14}$ Harvard Medical School,

Boston, MA, USA. ${ }^{15}$ Departments of Neurosurgery and Integrative Biology and Physiology, University of California Los Angeles, Los Angeles, CA, USA. ${ }^{16}$ Department of Psychiatry and Behavioural Neurosciences, McMaster University, Hamilton, ON, Canada. ${ }^{17}$ WELBIO-Walloon Excellence in Life Sciences and BIOtechnology, Metabolism and Nutrition Research Group, Louvain Drug Research Institute, UCLouvain, Université catholique de Louvain, Brussels,

Belgium. ${ }^{18}$ Basic and Clinical Neuroscience Department, Institute of Psychiatry, Psychology and Neuroscience, King's College London, London, UK. ${ }^{19}$ Nutrition Research Group, Research Institute of Biomedical and Health Sciences, University of Las Palmas de Gran Canaria, Las Palmas, Spain. ${ }^{20}$ Biomedical Research Center Network on Obesity and Nutrition (CIBERobn), Physiopathology of Obesity and Nutrition, Institute of Health Carlos III, Madrid, Spain. ${ }^{21}$ Université Paris-Saclay, UVSQ, Inserm, CESP, "DevPsy", 94807 Villejuif, France. ${ }^{22}$ Department of Epidemiology and Public Health, University College London, London, UK. ${ }^{23}$ Centre for Adolescent Health, Murdoch Children's Research Institute, Melbourne, VIC, Australia. ${ }^{24}$ Black Dog Institute, Sydney, NSW, Australia. ${ }^{25}$ James Cook University, Townsville, QLD, Australia. 凶email:wolf.marx@deakin.edu.au

\section{REFERENCES}

1. Jacka FN, O'Neil A, Itsiopoulos C, Opie R, Cotton S, Mohebbi M, et al. The SMILES trial: an important first step. BMC Med. 2018;16:1-3.

2. Nicolaou M, Colpo M, Vermeulen E, Elstgeest LE, Cabout M, Gibson-Smith D, et al. Association of a priori dietary patterns with depressive symptoms: a harmonised meta-analysis of observational studies. Psychol Med. 2020;50:1872-83.

3. Firth J, Marx W, Dash S, Carney R, Teasdale SB, Solmi M, et al. The effects of dietary improvement on symptoms of depression and anxiety: a meta-analysis of randomized controlled trials. Psychosom Med. 2019;81:265.

4. Jacka FN, O'Neil A, Opie R, Itsiopoulos C, Cotton S, Mohebbi M, et al. A randomised controlled trial of dietary improvement for adults with major depression (the 'SMILES'trial). BMC Med. 2017;15:1-13.

5. Parletta N, Zarnowiecki D, Cho J, Wilson A, Bogomolova S, Villani A, et al. A Mediterranean-style dietary intervention supplemented with fish oil improves diet quality and mental health in people with depression: a randomized controlled trial (HELFIMED). Nutritional Neurosci. 2019;22:474-87.

6. Francis HM, Stevenson RJ, Chambers JR, Gupta D, Newey B, Lim CK. A brief diet intervention can reduce symptoms of depression in young adults-a randomised controlled trial. PLoS ONE. 2019;14:e0222768.

7. Chatterton ML, Mihalopoulos C, O'Neil A, Itsiopoulos C, Opie R, Castle D, et al. Economic evaluation of a dietary intervention for adults with major depression (the "SMILES" trial). BMC Public Health. 2018;18:1-11.

8. Bot $M$, Brouwer IA, Roca M, Kohls E, Penninx BW, Watkins E, et al. Effect of multinutrient supplementation and food-related behavioral activation therapy on prevention of major depressive disorder among overweight or obese adults with subsyndromal depressive symptoms: the MooDFOOD randomized clinical trial. JAMA. 2019;321:858-68.

9. Vreijling SR, Penninx BW, Bot M, Watkins E, Owens $M$, Kohls $E$ et al. Effects of dietary interventions on depressive symptom profiles: results from the MooDFOOD depression prevention study. Psychol Med 2021;7:1-10.

10. Reynolds CF III, Thomas SB, Morse JQ, Anderson SJ, Albert S, Dew MA, et al. Early intervention to preempt major depression among older black and white adults. Psychiatr Serv. 2014:65:765-73.

11. Jacka FN. Nutritional psychiatry: where to next? EBioMedicine. 2017;17:24-29.
12. Malhi GS, Bell E, Bassett D, Boyce P, Bryant R, Hazell P, et al. The 2020 Royal Australian and New Zealand College of Psychiatrists clinical practice guidelines for mood disorders. Aust N Z J Psychiatry. 2021;55:7-117.

\section{AUTHOR CONTRIBUTIONS}

WM and FNJ drafted the manuscript. All authors provided feedback on the draft and approved of the final manuscript.

\section{FUNDING}

WM is currently funded by an Alfred Deakin Postdoctoral Research Fellowship and a Multiple Sclerosis Research Australia early-career fellowship. WM has previously received funding from the Cancer Council Queensland and university grants/ fellowships from La Trobe University, Deakin University, University of Queensland, and Bond University. WM has received industry funding and has attended events funded by Cobram Estate Pty. Ltd. WM has received travel funding from Nutrition Society of Australia, has received consultancy funding from Nutrition Research Australia and ParachuteBH, and has received speakers honoraria from The Cancer Council Queensland and the Princess Alexandra Research Foundation. MML is funded by a Deakin University PhD Scholarship and has received research support from BeFit Foods. MH is supported by an Australian Rotary Health PhD Scholarship and has received research support from The A2 Milk Company. HA is supported by a Deakin University Dean's Postdoctoral Research Fellowship. KW has previously received funding from Australian Research Council, National Health and Medical Research Council, and ChemGenex Pharmaceuticals. $A B$ and CMP have received research funding from Johnson \& Johnson for research on depression and inflammation, which included cellular work (2012-2018); moreover, CMP is funded by a Wellcome Trust strategy award to the Neuroimmunology of Mood Disorders and Alzheimer's Disease (NIMA) Consortium (104025), which is also funded by Janssen, GlaxoSmithKline, Lundbeck and Pfizer. The work presented in this paper is unrelated to this funding. $A B$ and $C M P$ are funded by the UK Medical Research Council (grants MR/ L014815/1, MR/J002739/1 and MR/N029488/1), the European Commission Horizon 2020 (grant SC1-BHC-01-2019) and the National Institute for Health Research (NIHR) Biomedical Research Centre at South London and Maudsley NHS Foundation Trust and King's College London. CMP is an NIHR Senior Investigator (2017-2025). KB is funded by an Irish Research Council postdoctoral fellowship and has received speakers honoraria from Yakult. JFC is supported by Science Foundation Ireland in the form of a Research Centre grant (SFI/12/RC/2273-P2), Joint Programming Initiative JPI-HDHL-NutriCog project 'AMBROSIAC' (15/JPHDHL/3270); Joint Programming Initiative HEALTHMARK: Metabolic HEALTH through nutrition, microbiota and tryptophan bioMARKers," 16/ERAHDHL/3362. (EU Horizon 2020 funding-DISCOvERIE (Development, dlagnosis and prevention of gender-related Somatic and mental COmorbiditiEs in iRrltable bowel syndrome in Europe), Swiss National Foundation Sinergia grant GUT-BRAIN; Irish Research Council; European Union's Horizon 2020 research and innovation programme under the Marie Skłodowska-Curie grant agreement number 797592 and 754535; Irish Research Council Postgraduate Scholarship GOIPG/2019/3198; GOIPG/2018/2560; Health Research Board (HRB) Grant number ILP-POR-2017-013; Institute for Scientific Information on Coffee and the Saks Kavanaugh Foundation. JFC received research support from Mead Johnson, Cremo, 4D Pharma, Pharmavite and Nutricia. He has been a consultant for Alkermes and Nestle, and has spoken at meetings sponsored by Mead Johnson, Abbott Nutrition, Roche, Ordesa and Neuraxpharm. GC is a funded investigator in APC Microbiome Ireland, a research centre funded by Science Foundation Ireland (SFI) (grant number $12 / \mathrm{RC} / 2273$ ). His research is currently supported by the Irish Health Research Board (HRB) (Grant number ILP-POR-2017-013). GC has received honoraria from Janssen, Probi and Apsen as an invited speaker, is in receipt of research funding from Pharmavite and Fonterra, and is a paid consultant for Yakult and Zentiva. J Firth is supported by a University of Manchester Presidential Fellowship (P123958) and a UK Research and Innovation Future Leaders Fellowship (MR/T021780/1), and has received honoraria/consultancy fees from Atheneum, ParachuteBH and Nirakara, independent of this work. JMC is currently funded by the Australian Research Council (DP190103081); the National Health and Medical Research Council (APP114333; APP1079102); the Waterloo Foundation; DNA Genotek; and Trajan Scientific and Medical. K-PS has received the following research grants related to this work: MOST109-2320-B-038-057-MY3, 109-2320-B-039-066, 110-2321-B-006-004, 110-2811B-039-507, 110-2320-B-039-048-MY2 and 110-2320-B-039-047-MY3 from the Ministry of Science and Technology, Taiwan; CMRC-CMA-2 from Higher Education Sprout Project by the Ministry of Education (MOE), Taiwan; CMU108-SR-106 from the China Medical University, Taichung, Taiwan; and CRS-108-048 and ANHRF109-31, 110-13 and 110-26 from An Nan Hospital, China Medical University, Tainan, Taiwan. K-PS has been a speaker and/or consultant for Johnson \& Johnson, AstraZeneca, Lundbeck, Eli Lilly, Merck, Pfizer, Servier, Otsuka, Excelsior Biopharma, Chen Hua Biotech, Nutrarex 
Biotech and Hoan Pharmaceuticals-all unrelated to this work. DM has received research support from Nordic Naturals and heckel medizintechnik $\mathrm{GmbH}$. He has provided unpaid consulting for Pharmavite LLC and Gnosis USA, Inc. He has received honoraria for speaking from the Massachusetts General Hospital Psychiatry Academy, Blackmores, Harvard Blog and PeerPoint Medical Education Institute, LLC. He has received royalties from Lippincott Williams and Wilkins for published book 'Natural medications for Psychiatric Disorders: considering the Alternatives'. He also works with the MGH Clinical Trials Network and Institute (CTNI), which has received research funding from multiple pharmaceutical companies and NIMH. FG-P is currently funded by research awards from the NINDS of National Institute of Health. He serves as a consultant and member of the Scientific Advisory Board of Nutrient Foods, LLC. J Foster received research funding from the National Sciences and Engineering Research Council of Canada (NSERC), and the Ontario Brain Institute, which is an independent non-profit corporation, funded partially by the Ontario Government. J Foster has received consultancy fees from Novozymes A/S and Rothmans, Benson \& Hedges, Inc. PDC is a senior research associate at FRS-FNRS (Fonds de la Recherche Scientifique), Belgium. He is supported by the Fonds Baillet Latour (Grant for Medical Research 2015), the Fonds de la Recherche Scientifique (FNRS, FRFS-WELBIO: WELBIOCR-2019C-02R) and ERC Starting Grant 2013 (336452-ENIGMO). PDC is the co-founder of A-Mansia Biotech SA and inventors on patent applications about the therapeutic use of Akkermansia muciniphila and its components. Diet and mental health research in the Thuret lab is funded by grants awarded by the Medical Research Council UK (MR/N030087/1 and MR/S00484X/1, the European Union's H2020 Joint Programming Initiative 'A Healthy Diet for a Healthy Life' and the Network of Centres of Excellence in Neurodegeneration (COEN). HS has previously received non-financial and financial support from CD investments VSL pharmaceuticals and is currently funded by an Alfred Deakin Postdoctoral Research Fellowship. AO'N is supported by a Future Leader Fellowship (\#101160) from the Heart Foundation Australia and Wilson Foundation. She has received research funding from the National Health \& Medical Research Council, Australian Research Council, University of Melbourne, Deakin University, Sanofi, Meat and Livestock Australia and Woolworths Limited, and Honoraria from Novartis. The Food \& Mood Centre has received funding from the
Fernwood Foundation, the A2 Milk Company and BeFit Foods. HS has previously received non-financial and financial support from CD investments VSL pharmaceuticals and is currently funded by an Alfred Deakin Postdoctoral Research Fellowship. FNJ is supported by an NHMRC Investigator Grant (\#1194982). She has received the following funding: (1) competitive Grant/Research support from the Brain and Behaviour Research Institute, the National Health and Medical Research Council (NHMRC), Australian Rotary Health, the Geelong Medical Research Foundation, the lan Potter Foundation, The University of Melbourne; (2) industry support for research from Meat and Livestock Australia, Woolworths Limited, the A2 Milk Company, BeFit Foods; (3) philanthropic support from the Fernwood Foundation, Wilson Foundation, the JTM Foundation, the Serp Hills Foundation, the Roberts Family Foundation, the Waterloo Foundation; and (4) travel support and speakers honoraria from SanofiSynthelabo, Janssen Cilag, Servier, Pfizer, Network Nutrition, Angelini Farmaceutica, Eli Lilly, Metagenics and The Beauty Chef. FNJ has written two books for commercial publication.

\section{COMPETING INTEREST}

KW, ST, AS-V, HA and TA declare no competing interests.

\section{ADDITIONAL INFORMATION}

Correspondence and requests for materials should be addressed to Wolfgang Marx.

Reprints and permission information is available at http://www.nature.com/ reprints

Publisher's note Springer Nature remains neutral with regard to jurisdictional claims in published maps and institutional affiliations. 